\section{(6) OPEN ACCESS}

- Additional material is published online only. To view please visit the journal online (http://dx.doi.org/10.1136/ gutjnl-2012-304351)

For numbered affiliations see end of article.

\section{Correspondence to} Dr Olivier Govaere, Translational Cell and Tissue Research, Department of Imaging and Pathology, University Hospitals Leuven, Minderbroedersstraat 12, Leuven B3000, Belgium; olivier.govaere@med. kuleuven.be

Received 16 December 2012 Revised 10 June 2013 Accepted 28 June 2013 Published Online First 19 August 2013

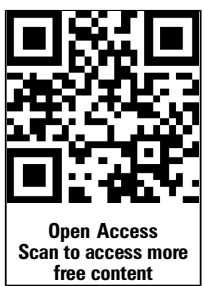

To cite: Govaere 0 , Komuta M, Berkers J, et al. Gut 2014;63:674-685.

\title{
Keratin 19: a key role player in the invasion of human hepatocellular carcinomas
}

Olivier Govaere, ${ }^{1}$ Mina Komuta, ${ }^{1}$ Johannes Berkers, ${ }^{1}$ Bart Spee, ${ }^{1}$ Carl Janssen, ${ }^{1}$ Francesca de Luca, ${ }^{2}$ Aezam Katoonizadeh, ${ }^{1}$ Jasper Wouters, ${ }^{1,3}$ Léon C van Kempen, ${ }^{4}$ Anne Durnez, ${ }_{1}^{1}$ Chris Verslype, ${ }^{5}$ Joery De Kock, ${ }^{6}$ Vera Rogiers, ${ }^{6}$ Leo A van Grunsven, ${ }^{7}$ Baki Topal, ${ }^{8}$ Jacques Pirenne, ${ }^{9}$ Hugo Vankelecom, ${ }^{3}$ Frederik Nevens, ${ }^{5}$ Joost van den Oord, ${ }^{1}$ Massimo Pinzani, ${ }^{10}$ Tania Roskams ${ }^{1}$

\section{ABSTRACT}

Objective Keratin (K)19, a biliary/hepatic progenitor cell (HPC) marker, is expressed in a subset of hepatocellular carcinomas (HCC) with poor prognosis. The underlying mechanisms driving this phenotype of K19-positive HCC remain elusive.

Design Clinicopathological value of K19 was compared with EpCAM, and $\alpha$-fetoprotein, in a Caucasian cohort of 242 consecutive patients (167 surgical specimens, 75 needle biopsies) with different underlying aetiologies. Using microarrays and microRNA profiling the molecular phenotype of K19-positive HCCs was identified. Clinical primary HCC samples were submitted to in vitro invasion assays and to side population analysis. HCC cell lines were transfected with synthetic siRNAs against KRT19 and submitted to invasion and cytotoxicity assays. Results In the cohort of surgical specimens, K19 expression showed the strongest correlation with increased tumour size $(p<0.01)$, decreased tumour differentiation $(p<0.001)$, metastasis $(p<0.05)$ and microvascular invasion $(p<0.001)$. The prognostic value of $\mathrm{K} 19$ was also confirmed in a set of 75 needle biopsies. Profiling showed that K19-positive HCCs highly express invasion-related/metastasis-related markers (eg, VASP, TACSTD2, LAMB1, LAMC2, PDGFRA), biliary/HPC markers (eg, CD133, GSTP1, NOTCH2, JAG1) and members of the miRNA family 200 (eg, miR-141, miR200c). In vitro, primary human K19-positive tumour cells showed increased invasiveness, and reside in the chemoresistant side population. Functionally, K19/KRT19 knockdown results in reduced invasion, loss of invadopodia formation and decreased resistance to doxorubicin, 5-fluorouracil and sorafenib.

Conclusions Giving the distinct invasive properties, the different molecular profile and the poor prognostic outcome, K19-positive HCCs should be considered as a seperate entity of HCCs.

\section{INTRODUCTION}

The expression of keratin (K)19, a marker for cholangiocytes, hepatic progenitor cells (HPCs) and early hepatoblasts, has been linked with a poor prognosis for patients diagnosed with hepatocellular carcinoma (HCC). ${ }^{1}$ Over the past years, many Eastern studies, mainly on HBV-infected patients, associated the occurrence of K19 with clinicopathological features, such as metastasis, poor tumour differentiation, tumour recurrence after

\section{Significance of this study}

What is already known on this subject?

- Keratin (K)19, a biliary/hepatic progenitor cell (HPC) marker, is expressed in a subset of hepatocellular carcinomas (HCC) with poor prognosis, and is thought to reflect the cell of origin.

- Over the past years, many Eastern studies, mainly on HBV infected patients, have associated the occurrence of K19 with clinicopathological features, such as metastasis, poor tumour differentiation, tumour recurrence after resection and radiofrequency ablation, as well as poor overall survival.

- In 2006, our group was the first to describe the clinicopathological and prognostic relevance of K19 in a Caucasian series of HCCs as it was linked with a high recurrence after transplantation.

\section{What are the new findings?}

- In this prospective Caucasian series of 242 consecutive HCCs, K19 expression was most strongly correlated with clinicopathological parameters, in comparison with EPCAM and $\alpha$-fetoprotein, including increased tumour size, decreased tumour differentiation, metastasis and microvascular invasion.

- Thorough molecular profiling (including mRNA and microRNA arrays) revealed a novel regulatory network associated with K19 expression which, in turn, intersected with pathways controlling cytoskeleton dynamics and cellular motility. microRNA overexpression confirmed a role for several novel candidate microRNAs (eg, miR-141, miR-200c) in modulating K19-associated genes.

- We further demonstrated in vitro, using primary human HCC samples, that K19 expression confers an invasive phenotype. K19 expression was also associated with chemoresistance, which was demonstrated using the side population cell isolation technique and cytotoxic assays.

- Our findings demonstrate functional consequences of K19 expression, since KRT19 knockdown significantly reduced HCC invasive ability in vitro and compromised the formation of invadopodia. 
Significance of this study

\section{How might it impact on clinical practice in the} foreseeable future?

- Our data sheds new light on the mechanisms underlying the poor prognostic K19 phenotype, and strongly supports the contention that K19-positive HCC should be diagnosed and treated as a separate entity of HCCs.

resection and radiofrequency ablation, as well as poor overall survival. ${ }^{2-6}$ In 2006, our group was the first to describe the clinicopathological and prognostic relevance of K19 in a Caucasian series of HCCs as it was linked with a high recurrence after transplantation. ${ }^{7}$ Subsequently, we started a prospective study on a Caucasian series of 242 consecutive HCC samples with a different range of aetiologies and performed a detailed evaluation of the clinicopathological relevance of K19.

Although various publications describe the prognostic relevance of K19 in HCCs, it remains unclear why these particular tumours behave more aggressively. One hypothesis finds its explanation in the possible cell of origin. ${ }^{8}$ Approximately $80 \%$ of HCCs arise on a background of long-lasting chronic liver disease, where there is an extensive activation of the HPC compartment. Bipotential HPCs have the capacity to differentiate into hepatocytes or cholangiocytes to facilitate liver regeneration, but their activation and proliferation also provide a potential source for carcinogenesis. In humans, the description of primary hepatic carcinomas with mixed hepatocellular/cholangiocellular features and HCCs displaying progenitor/stemness features, at least supports the idea that some primary liver cancers arise from HPCs. ${ }^{9}$ HPCs are thought to be resilient and to be able to survive in a chronic diseased liver, an environment full of inflammation, oxidative stress and necrosis, due to their high expression of ATP-binding cassette (ABC) transporters. ${ }^{10}$ $\mathrm{ABC}$ transporters protect the cell by actively transporting a wide variety of substrates (including xenobiotics) across the cell membrane, using ATP during the process, but also contribute to multidrug chemoresistance, rendering tumour cells resistance to systemic therapies. ${ }^{11}$ Several of these ABC transporters are reported to colocalise with K19 expression in HCCs, suggesting that K19-positive HCCs and HPCs share similar protective mechanisms. ${ }^{10}$

In this present study, we assess the clinicopathological relevance of $\mathrm{K} 19$ in a Caucasian series of 242 consecutive HCC samples in comparison with other biliary/HPC markers, epithelial cell adhesion molecule (EpCAM) and $\alpha$-fetoprotein (AFP), and we also unravel the underlying molecular phenotype of K19-positive HCCs, using microarrays and microRNA (miRNA/miR) profiling. Several of these miRNAs were shown to contribute to the K19-positive phenotype. Trying to further elude the more aggressive behaviour of these tumours, clinical human HCC samples were submitted to in vitro invasion assays and to side population (SP) cell isolations, a technique used to isolate a chemoresistant subpopulation based on the ability of ABC transporters to efflux Hoechst 33342. Finally, the functional role of K19 and its effect to associated genes was evaluated in relationship to invasion and resistance to cytotoxic agents.

\section{METHODS}

\section{Patient selection}

The diagnosis of HCC was based on WHO criteria. Totally, 242 formalin-fixed paraffin-embedded (FFPE) liver biopsies from 242 consecutive patients with HCC, treated at the University Hospitals Leuven between 2003 and 2008, were included. The 242 samples were divided into two groups, consisting of 167 surgical specimens and 75 needle biopsies. In case of multiple nodules per patient (eg, satellite nodules in explant livers), the largest HCC nodule was selected as representative sample for the patient. For the surgical specimens, the tumour size was measured macroscopically after the removal of the sample; tumour differentiation and microvascular invasion was graded based on histopathological evaluation. Microvascular invasion was scored in the tumour and in the surrounding tissue of the tumour. Positivity for metastasis was established using histopathological evaluation and/or based on available clinical data (126 of the 167). Needle biopsies were taken for diagnostic purpose or before a surgical procedure (eg, radiofrequency ablation) and were scored for microvascular invasion and differentiation grade similar to the surgical specimens. Metastasis was analysed solely based on the available clinical data (17 of the 75). Tumour size for the needle biopsies was not established.

K19, EPCAM and AFP expression was semiquantitatively assessed by means of immunohistochemistry on consecutive FFPE slides. Samples were considered positive for a marker when expression was seen, using a cut-off value of 5\% immunohistochemical positivity to exclude false positives, as previously described. ${ }^{7}$ Contingency analysis was done using the Fisher's Exact test and the unpaired t test with STATVIEW 5.0.1 software (SAS Institute, Cary, North Carolina, USA). The study was approved by the ethical committee of the University Hospitals Leuven.

\section{Immunohistochemistry and in situ hybridisation}

Of the 242 HCC samples stained for K19 (1/25; Dako, Glostrup, Denmark), EPCAM (1/50; Dako) and AFP (1/500; Dako), a subset (K19-positive and -negative $n=12$ per group) was selected as a validation set for some selected targets: laminin (1/50; Novocastra, NewCastle, UK), VASP (1/100; Sigma-Aldrich, St Louis, Missouri, USA), PDGFRA (1/50; Abcam, Cambridge, UK) and TACSTD2 (1/20; Abcam). Immunohistochemistry/immunocytochemistry was performed as previously described. ${ }^{10}{ }^{12}$ In situ hybridisation was performed with DIG-labelled probes against hsa-miR-141 and hsa-miR-200c (Exiqon, Vedbaek, Denmark) as reported previously. ${ }^{13}$

\section{Microarray analysis}

Microarray analysis was performed on 139 HCC samples (GSE1898 and GSE4024). ${ }^{14}{ }^{15}$ Gene expression profiles were generated at the Advanced Technology Centre (National Cancer Institute, USA). Data transformation and normalisation of gene expression was performed as previously reported. Association with clinicopathological features and statistical analysis are described in online supplementary file 1. Gene Set Enrichment Analysis was used to statistically analyse the overlap of KRT19 correlated genes with previous published gene expression signatures (see online supplementary files 2 and 3 ). $16 \quad 17$ Additionally, STRING analysis was performed to predict possible interactions, either direct (physical) or indirect (functional) associations. ${ }^{18}$ 
Quantitative real-time PCR (qPCR)

qPCR Was performed on a subset of 12 frozen HCC resection specimens, K19-positive $(n=6)$ or -negative $(n=6)$, diagnosed at the University Hospitals Leuven Belgium. RNA extraction, cDNA synthesis and qPCR analysis were performed as previously described. ${ }^{12}$ Overview of custom-designed primer sequences can be found in online supplementary file 4 .

\section{Invasion assay and SP analysis}

Tissue samples obtained from patients diagnosed with HCC (K19-positive $n=6$, negative $n=6$ ) at the University Hospitals Leuven between 2008 and 2011 were cryopreserved postsurgery and histopathologically characterised. Samples were dissociated using LiberaseBlendzyme3 (Roche, Basel, Switzerland; 0.8 Wunsch unit $/ \mathrm{mL}, 1.5 \mathrm{~h}, 37^{\circ} \mathrm{C}$ ). After filtration and gradient centrifugation, the cells were resuspended in Advanced Dulbecco's Modified Eagle's Medium supplemented with L-Glutamine (Invitrogen, Carlsbad, California, USA) and submitted to a QCM ECMatrix Cell Invasion Assay (Millipore, Billerica, Massachusetts, USA; $8 \mu \mathrm{m}$ ). Fetal bovine serum (Invitrogen) was used as chemoattractant. After $48 \mathrm{~h}$, invaded cells were detected by crystal violet stain and quantified at an optical density (OD) of $560 \mathrm{~nm}$. Parallel, invaded cells were immunostained after $48 \mathrm{~h}$ for $\mathrm{K} 19$, as described above.

To assess active efflux and SP phenotype, dissociated HCC cells were incubated with or without the transport blocker verapamil (100 $\mu \mathrm{M}$; Sigma-Aldrich), prior to the Hoechst33342 incubation $(5 \mu \mathrm{g} / \mathrm{mL}$; Sigma-Aldrich) as previously reported. Propidium iodide $(2 \mu \mathrm{g} / \mathrm{mL}$; Sigma-Aldrich) was added to exclude dead cells. Cell suspensions were analysed using a FACSArialI (BDBiosciences). A detailed description of the dissociation protocol and SP method can be found in online supplementary file 4.

\section{In vitro transient knockdown of KRT19/K19}

Human HCC cell lines HepG2, Huh-7D12, PLC/PRF/5 (ECACC, Salisbury, UK) were transfected using ON-TARGETplus siRNA against KRT19 or ON-TARGETplus Non-targeting siRNA at a final concentration of $25 \mathrm{nM}$ (Dharmacon, Lafayette, Colorado, USA), using Lipofectamine RNAiMAX Transfection Reagent (Invitrogen). After $24 \mathrm{~h}$, transfected cells were submitted to a QCM ECMatrix Cell Invasion Assay and a MTT Cell Growth Assay (Millipore) for $48 \mathrm{~h}$. Colorimetric reading of the Invasion Assay was done at OD560 $\mathrm{nm}$; the MTT Assay at OD570 $\mathrm{nm}$ using a reference point of $630 \mathrm{~nm}$. Results of invasion were normalised to the proliferation results. In parallel, cells were transfected and harvested after $72 \mathrm{~h}$ for total mRNA extraction and qPCR analysis.

\section{In vitro stable knockdown of KRT19/K19}

Huh-7D12 cells were transduced using SMARTvector 2.0 Human Lentiviral shRNA Particles KRT19, hCMV-TurboGFP or SMARTvector 2.0 Empty Vector Control Particles, according to supplier's protocol (Dharmacon). Cells were submitted to the QCM Gelatin Invadopodia Assay Green (Millipore) according to supplier's protocol. After $24 \mathrm{~h}$, cells were fixated in acetone for $10 \mathrm{~min}$ and stained for Cytokeratin Wide Spectrum Screening (DAKO) for $60 \mathrm{~min}$, followed by an incubation with Alexa Fluor 568 Goat Anti-Rabbit (1/100, Invitrogen) for 30 min and counterstaining with DAPI (Invitrogen).

Additionally, Huh-7D12 cells with stable KRT19 knockdown or control vector were treated with doxorubicin $(1 \mu \mathrm{M})$, 5-fluorouracil $(1 \mathrm{mM})$ and sorafenib $(5 \mu \mathrm{M})$ (Selleckchem,
Texas, USA), supplemented to the culture medium for $72 \mathrm{~h}$ and submitted to a MTT assay in comparison with untreated cells.

\section{MicroRNA profiling and In vitro modulation}

Genome-wide $\mathrm{RT}^{2}$ miRNA PCR Arrays (384-well based; SABiosciences, Frederick, Maryland, USA) were performed on frozen HCC liver biopsies $(n=12)$, according to supplier's protocol. Human HCC cell lines were characterised using RT $^{2}$ miRNA qPCR Primer Assays (SABiosciences). Normalisation of the cycle threshold data was done to reference small RNA (U6, SNORD44, 47 and 48$)$, fold-change was calculated based on the $2^{(-\Delta \Delta \mathrm{C} t)}$ method. The PLC/PRF/5 cell line was transfected $(n=3)$ with miRIDIAN Dharmacon microRNA Mimics (miR-141, miR-200c, negative control) at a final concentration of $2 \mathrm{nM}$, using DharmaFECT4 Transfection Reagent (Dharmacon), according to supplier's protocol. Cells were harvested after $48 \mathrm{~h}$ prior to total mRNA extraction.

\section{Statistical analysis}

Statistical analysis for qPCR, MTT assays and invasion assays was performed using the non-parametric Kruskal-Wallis and the Man-Whitney U test, with Graphpad Prism 5.02 (GraphPad Software, La Jolla, California, USA). All analyses were twotailed. In all cases, $\mathrm{p}<0.05$ was considered significant.

\section{RESULTS}

\section{Occurrence and clinicopathological relevance of HCC} prognostic markers

After our previous retrospective study, demonstrating an association between K19 expression and HCC recurrence after transplantation, we started a prospective study to further investigate 'stemness' features in HCC and the role of K19. ${ }^{7}$ Biliary/HPC markers K19, EPCAM and AFP were compared and linked with clinicopathological features in a cohort of 242 consecutive patients, consisting of 167 surgical specimens and 75 needle biopsies. In 167 surgical specimens, $11.38 \%$ showed K19 immunopositivity $(\mathrm{n}=19), 14.97 \%$ EPCAM positivity $(\mathrm{n}=25)$ and $10.78 \%$ AFP positivity $(n=18)$ (figure 1A). Only $2.99 \%(n=5)$ showed simultaneous positivity for all three markers. In total, $62.87 \%$ of the HCCs were negative for all three markers $(\mathrm{n}=105)$. Immunohistochemically, K19 showed a submembranous and EPCAM a membranous localisation, whereas AFP showed a diffuse cytoplasmic signal (figure 1B-D). K19 expression was significantly correlated with increased tumour size $(\mathrm{p}<0.01)$, decreased tumour differentiation $(\mathrm{p}<0.001)$, metastasis $(\mathrm{p}<0.05)$ and microvascular invasion $(\mathrm{p}<0.001)(($ table 1$)$. AFP expression significantly correlated with decreased tumour differentiation $(p<0.001)$ and microvascular invasion $(\mathrm{p}<0.001)$, whereas EPCAM only correlated with microvascular invasion $(p<0.05)$. The clinicopathological relevance of K19 expression was also significant in a cohort of 75 needle biopsies (table 1). K19 expression was seen in $20 \%$ of the samples $(n=15)$ and was significantly linked with decreased tumour differentiation $(\mathrm{p}<0.001)$, metastasis $(\mathrm{p}<0.05)$ and microvascular invasion $(\mathrm{p}<0.01)$.

\section{Molecular phenotype of K19-positive HCCs}

In order to unravel the molecular/transcriptomic background of K19-positive HCCs, and to discover K19-associated pathways, we used a microarray database of 139 HCC samples. Similar to K19 protein expression, KRT19 mRNA expression was significantly associated with clinicopathological parameters and was an independent predictor for tumour recurrence (Log rank test, $\mathrm{p}=0.007$; HR 1.70) (see online supplementary file 1). 


\section{A}

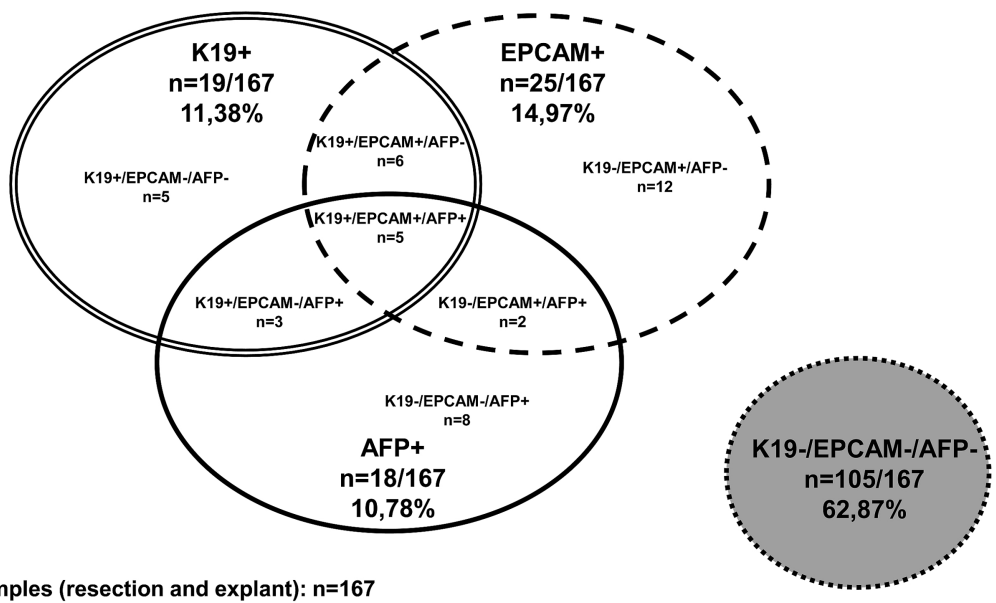

Surgical HCC samples (resection and explant): $n=167$
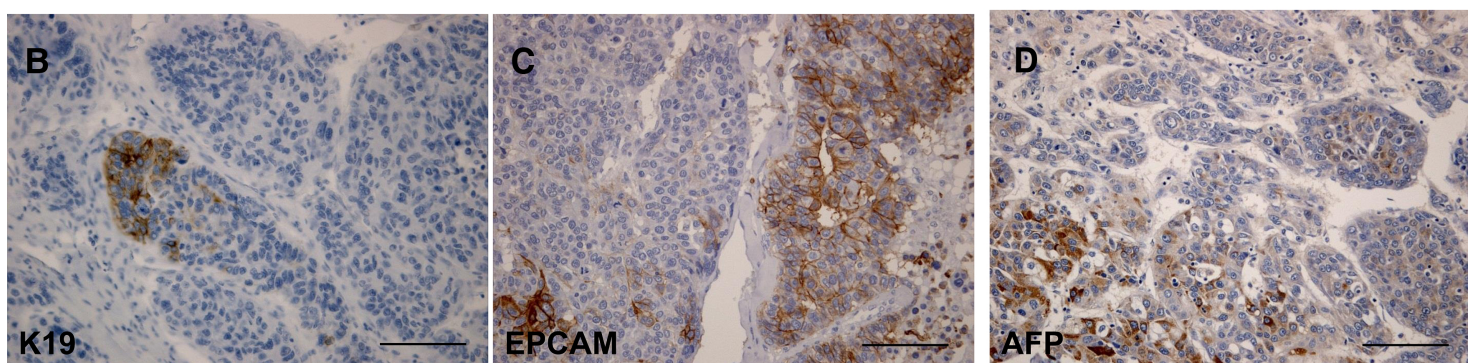

Figure 1 Occurrence and clinicopathological relevance of K19, EPCAM and $\alpha$-fetoprotein (AFP) in hepatocellular carcinomas (HCCs). (A) Out of the 167 surgical HCC specimens $11.38 \%$ showed K19 positivity $(n=19), 14.97 \%$ EPCAM positivity $(n=25)$, and 10.78\% AFP positivity $(n=18)$. Only $2.99 \%(n=5)$ showed simultaneous positivity for all three markers. (B-D) Immunohistochemistry of a HCC sample positive for K19 (B), EPCAM (C) and AFP (D). K19 showed a submembranous positivity and EPCAM a membranous one, whereas, AFP showed a diffuse cytoplasmic positivity. Scale bars $250 \mu \mathrm{m}$.

Out of 21329 genes quantified by microarray, 132 genes correlated positively, and 203 genes negatively with KRT19 expression (see online supplementary file 2). Gene Set Enrichment Analysis of the genes positively correlated with KRT19 showed a strong correlation with several other cancer-related datasets, including 'poor survival HCC subtype', 'proliferation HCC subtype', 'subtype S1 signature with aberrant Wnt activation' (see online supplementary file 3). ${ }^{16}$ Genes negatively correlated with KRT19 showed a significant overlap with liver-/hepatocytespecific genes and genes downregulated in malignant HCC subtypes (see online supplementary file 3). Additionally, STRING analysis was performed to predict protein-protein interactions among the genes that were positively correlated with K19 expression, in order to gain insight into the molecular mechanisms underlying the aggressive nature of K19-positive HCCs. ${ }^{18}$ K19 was clearly linked with a node around the gene vasodilatorstimulated phosphoprotein (VASP) and genes involved in the extracellular matrix (ECM) (eg, VCL, LAMB1, LAMC2, ITGA3, ITGB6) (see online supplementary file 3). qPCR Validation of KRT19-correlated genes and selected candidates, confirmed that K19-positive HCCs express high levels of other biliary/HPCs cell markers (eg, KRT7, CD133, GSTP1) in addition to KRT19, when compared with K19-negative HCCs (figure 2A). Moreover, we observed strong upregulation of members of the Notch signalling pathway (eg, NOTCH1, NOTCH2, NOTCH3, JAG1, JAG2), reduced expression of hepatocyte-specific genes (eg, HNF4A, $A B C C 2)$ and induction of invasion-related/metastasis-related markers (eg, CTBP2, S100A6, VASP, FAM57A, TACSTD2, $A N X A 3, R A B 25, R A B 27 B)$. By contrast, the metastasis suppressor MTSS1 was downregulated. We also observed alterations in mRNAs encoding cytoskeletal regulators (upregulation of
IQGAP1 and downregulation of IQGAP2) and ECM components (upregulation of $L A M B 1$ and LAMC2) in K19-positive tumours. Immunohistochemistry revealed that a large fraction of K19-positive HCCs exhibited abundant cytoplasmic laminin, as was observed in the basement membrane around HPCs and in individual HPCs in the neighbouring cirrhotic tissue (figure 2B). Additionally, K19-positive HCCs showed an intense submembranous positivity for VASP and a membranous positivity for platelet-derived growth factor receptor $\alpha$ (PDGFRA). Tumourassociated calcium signal transducer 2 (TACSTD2/TROP2) showed a membranous reactivity in a small portion of the HCCs that were poorly differentiated and K19-positive. Morphologically, TACSTD2-positive tumour cells showed a more progenitorlike phenotype with a high cell density and scant cytoplasm. In the non-tumoural surrounding tissue, TACSTD2, VASP and laminin were found to be mainly expressed by the HPCs and the smaller bile ducts, whereas, the larger bile ducts showed a variable, focal positivity for these proteins. PDGFRa, by contrast, was expressed by all the biliary epithelial cells and the HPCs (figure 2B, online supplementary file 5).

\section{A functional role for K19 in invasion and chemoresistance}

In view of the strong association between K19 expression and microvascular invasion, and the high expression of invasionrelated genes, we evaluated the invasive potential of K19-positive HCC cells in vitro. Dissociated primary human HCCs, selected for K19 immunopositivity, were used (figure 3A). K19 positivity was first assessed in FFPE tissue samples and subsequently in cytospins processed from dissociated cells. Both K19-positive and negative dissociated tumour cells were subjected to an ECM cell invasion assay. Only invasive cells can migrate through the 
Table 1 Correlation of K19, EPCAM and $\alpha$-fetoprotein (AFP) expression with clinicopathological features of surgical hepatocellular carcinoma (HCC) samples (resection and explant) obtained from 167 patients

\begin{tabular}{|c|c|c|c|c|c|c|}
\hline & K19-positive HCCs & K19-negative HCCs & EPCAM-positive HCCs & EPCAM-negative HCCs & AFP-positive HCCs & AFP-negative HCCs \\
\hline Immunopositivity & $11.38 \%(19 / 167)$ & $88.62 \%(148 / 167)$ & $14.97 \%(25 / 167)$ & $85.03 \%(142 / 167)$ & $10.78 \%(18 / 167)$ & $89.22 \%(149 / 167)$ \\
\hline Age (years) (mean) & 60.05 (ns) & 62.04 (ns) & 58.2 (ns) & 62.45 (ns) & 58.94 (ns) & 62.16 (ns) \\
\hline \multicolumn{7}{|l|}{ Gender } \\
\hline Male & $63.16 \%(12 / 19 ; \mathrm{ns})$ & $74.32 \%$ (110/148; ns) & $68 \%(17 / 25 ; n s)$ & $63.94 \%$ (105/142; ns) & $77.78 \%$ (14/18; ns) & $72.48 \%$ (108/149; ns) \\
\hline Female & $36.84 \%$ (7/19; ns) & $25.68 \%$ (38/148; ns) & $32 \%$ (8/25; ns) & $26.06 \%$ (37/142; ns) & $22.22 \%$ (4/18; ns) & $27.52 \%$ (41/149; ns) \\
\hline \multicolumn{7}{|l|}{ Aetiology } \\
\hline HBV & $15.79 \%$ (3/19; ns) & $7.43 \%$ (11/148; ns) & $20 \%$ (5/25; ns) & $6.34 \%(9 / 142 ; \mathrm{ns})$ & $22.22 \%$ (4/18; ns) & $6.04 \%$ (9/149; ns) \\
\hline $\mathrm{HCV}$ & $5.26 \%$ (1/19; ns) & $29.73 \%$ (44/148; ns) & $12 \%$ (3/25; ns) & $29.58 \%$ (42/142; ns) & $22.22 \%$ (4/18; ns) & $27.52 \%$ (41/149; ns) \\
\hline $\mathrm{HBV}+\mathrm{HCV}$ & $0 \%$ (0/19; ns) & $2.70 \%$ (4/148; ns) & $0 \%(0 / 25 ; \mathrm{ns})$ & $2.82 \%$ (4/142; ns) & $0 \%(0 / 18 ; \mathrm{ns})$ & $2.69 \%$ (4/149\%; ns) \\
\hline ALD & $10.52 \%(2 / 19 ; n s)$ & $16.22 \%$ (24; ns) & $16 \%(4 / 25 ; n s)$ & $15.49 \%(22 / 142 ; n s)$ & $16.67 \%$ (3/18; ns) & $15.44 \%$ (23/149; ns) \\
\hline ALD+viral infection & $5.26 \%$ (1/19; ns) & $2.03 \%$ (3/148; ns) & $4 \%(1 / 25 ; \mathrm{ns})$ & $2.11 \%$ (3/142; ns) & $0 \%(0 / 18 ; \mathrm{ns})$ & $2.01 \%$ (3/149; ns) \\
\hline Other & $36.84 \%$ (7/19; ns) & $27.70 \%$ (41/148; ns) & $36 \%$ (9/36; ns) & $27.46 \%$ (39/142; ns) & $27.78 \%$ (5/18; ns) & $30.2 \%$ (45/149; ns) \\
\hline Unknown & $26.32 \%$ (5/19; ns) & $14.19 \%(21 / 148 ; n s)$ & $12 \%$ (3/25; ns) & $16.2 \%(23 / 142 ; n s)$ & $11.11 \%(2 / 18 ; \mathrm{ns})$ & $16.11 \%(24 / 149 ; n s)$ \\
\hline Microvascular Invasion & $100 \%(19 / 19 ; p=0.0001)$ & $52.03 \%$ (77/148; ns) & $76 \%(19 / 25 ; p=0.0423)$ & $54.23 \%$ (77/142; ns) & $100 \%(18 / 18 ; p=0.0001)$ & $52.35 \%$ (78/149; ns) \\
\hline Metastasis & $83.33 \%(10 / 12 ; p=0.0207)$ & $48.25 \%$ (55/114; ns) & $58.82 \%(10 / 17 ; \mathrm{ns})$ & $50.46 \%(55 / 109 ; \mathrm{ns})$ & $76.92 \%$ (10/13; ns) & $48.67 \%$ (55/113; ns) \\
\hline Tumour size (cm. mean) & $6.77(p=0.0077)$ & 4.5 & 5.27 (ns) & 4.72 & 4.92 (ns) & 4.79 \\
\hline \multicolumn{7}{|l|}{ Differentiation grade } \\
\hline Well & $5.26 \%(1 / 19)$ & $45.27 \%(67 / 148)$ & $32 \%(8 / 25)$ & $42.25 \%(60 / 142)$ & $5.56 \%(1 / 18)$ & $44.97 \%(67 / 149)$ \\
\hline Moderate & $36.84 \%(7 / 19)$ & $36.84 \%(54 / 148)$ & $28 \%(7 / 25)$ & $38.03 \%(54 / 142)$ & $38.89 \%(7 / 18)$ & $36.24 \%(54 / 149)$ \\
\hline Poorly & $57.89 \%(11 / 19 ; p=0.0001)$ & $18.24 \%$ (27/148; ns) & $40 \%$ (10/25; ns) & $19.72 \%(28 / 142 ; n s)$ & $55.56 \%(10 / 18 ; p=0.0004)$ & $18.79 \%(28 / 149 ; \mathrm{ns})$ \\
\hline \multicolumn{7}{|l|}{ Cirrhosis } \\
\hline No & $31.58 \%$ (6/19;ns) & $23.65 \%$ (35/148; ns) & $44 \%(11 / 25 ; n s)$ & $21.13 \%$ (30/142; ns) & $27.78 \%$ (5/18; ns) & $24.16 \%$ (36/149; ns) \\
\hline Yes & $68.42 \%$ (13/19;ns) & $75 \%$ (111/148; ns) & $56 \%(14 / 25 ; n s)$ & $77.46 \%$ (110/142; ns) & $72.22 \%$ (13/18. ns) & $74.5 \%$ (111/149; ns) \\
\hline Unknown & $0 \%$ (0/19; ns) & $1.35 \%(2 / 148 ; n s)$ & $0 \%(0 / 25 ; \mathrm{ns})$ & $1.41 \%(2 / 142 ; n s)$ & $0 \%(0 / 18 ; \mathrm{ns})$ & $1.34 \%(2 / 149 ; \mathrm{ns})$ \\
\hline
\end{tabular}

Correlation of K19 expression with clinicopathological features of needle biospies obtained from 75 patients.

\begin{tabular}{|c|c|c|}
\hline & K19 positive HCCs & K19 negative HCCs \\
\hline Immunopositivity & $20 \%(15 / 75)$ & $80 \%(60 / 75)$ \\
\hline Age (years) (mean) & 63.93 (ns) & 64.13 (ns) \\
\hline \multicolumn{3}{|l|}{ Gender } \\
\hline Male & $66.67 \%(10 / 15 ; \mathrm{ns})$ & $73.33 \%$ (44/60; ns) \\
\hline Female & $33.33 \%$ (5/15; ns) & $26.67 \%$ (16/60; ns) \\
\hline Microvascular Invasion & $66.67 \%(10 / 15 ; p=0.007)$ & $21.67 \%(13 / 60 ; n s)$ \\
\hline Metastasis & $100 \%(5 / 5 ; p=0.0048)$ & $25 \%$ (3/12; ns) \\
\hline
\end{tabular}




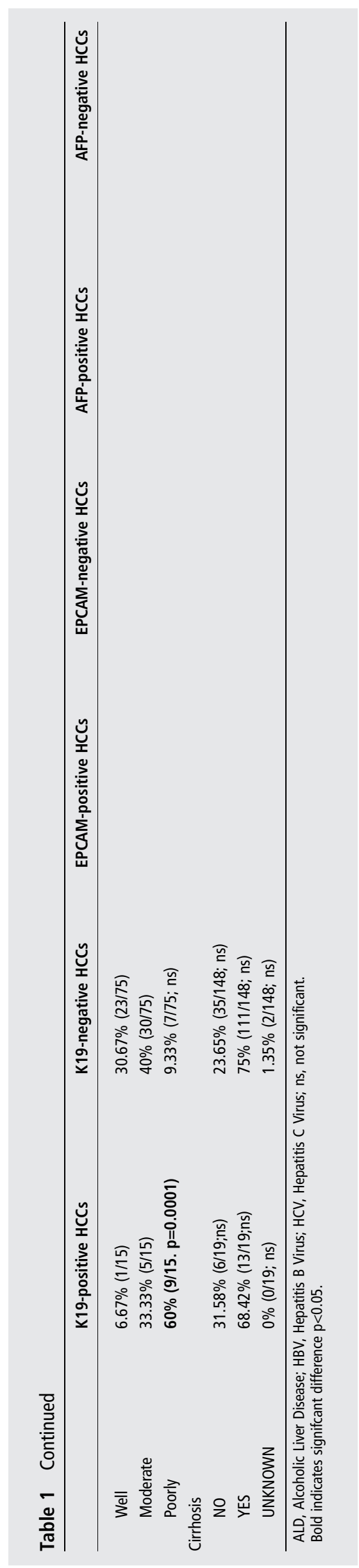

ECM layer and cling to the bottom of a polycarbonate membrane. After 48 h, cells from human primary K19-positive HCCs showed a higher invasiveness than cells from K19-negative HCCs $(\mathrm{p}<0.01 ; \mathrm{n}=12)$. Interestingly, especially the K19-positive tumour cells invaded through the basement membrane, as found by immunostaining (figure 3A).

To investigate the functional role of $\mathrm{K} 19$, we performed siRNA knockdown experiments in three different HCC cell lines: the Huh-7D12 cell line was characterised as a cell line showing 100\% K19 positivity, whereas HepG2 cells showed a weak focal positivity in about $20 \%$ of the cells and the PLC/ $\mathrm{PRF} / 5$ cell line was negative (figure $3 \mathrm{~B}$ ). Using an invasion assay, transient knockdown of KRT19 reduced the invasiveness of Huh-7D12 after $72 \mathrm{~h}$ by $34.7 \%(\mathrm{p}<0.01)$ when compared with the negative control (scrambled siRNA). By contrast, no significant differences in invasiveness were found in HepG2 and PLC/ $\mathrm{PRF} / 5$ (figure 3B). Given the altered invasive potential of Huh-7D12, we further examined the effect of KRT19 knockdown on the expression of genes identified above. A significant reduction in CD133, VASP, LAMB1, IQGAP1, ROCK2 $(\mathrm{p}<0.05)$ and NOTCH2, JAG1, RAB25 ( $\mathrm{p}<0.01)$ expression was observed (figure $3 \mathrm{~B}$ ). No significant differences were found in the expression levels of ANXA3, S100A6, HNF4A, PDGFRA, IQGAP2, LAMC2, CTBP2 and TACSTD2. In keeping with the results of the migration assay, stable transduction of Huh-7D12 cells with the KRT19 shRNA construct resulted in decreased formation of invadopodia, as tested in a GFP-conjugated gelatin degradation assay (figure $3 \mathrm{C}$ ).

In HCC cell lines, SP technique, a method based on the functional ability of ABC transporters to efflux Hoechst33342, has proven to be useful to isolate a chemoresistant cell fraction with HPC features. ${ }^{19}$ In this study, we apply the SP technique to further functionally characterise K19-positive HCCs in comparison with K19-negative HCCs. Results indicate that the SP comprised a larger proportion in K19-positive HCCs $(10.2 \%$ $\pm 4.1 ; \mathrm{n}=6)$ than in K19-negative HCCs $(3.3 \% \pm 2.4 ; \mathrm{n}=6)$ (figure 4A,B). Immunohistochemistry of cytospins from sorted SP cells and bulk tumour cells (main population or MP) showed that all K19-positive cells were located in the SP (figure 4C). Additionally, impairing KRT19 expression in Huh-7D12 cells by stable transduction with KRT19 shRNA constructs, rendered the cells more sensitive to treatments with either $1 \mu \mathrm{M}$ doxorubicin, $1 \mathrm{mM}$ fluorouracil or $5 \mu \mathrm{M}$ sorafenib than Huh-7D12 cells transduced with the control construct $(\mathrm{p}<0.05 ; \mathrm{t}=72 \mathrm{~h}, \mathrm{n}=3)$. The effect was measured compared with untreated cells, using a MTT assay, and displayed as \% viable cells (figure 4D).

\section{MicroRNA 200 family is linked with KRT19/K19 expression}

Finally, an alternative profiling approach based on microRNA (miRNA/miR) expression was used to identify the K19-positive HCC phenotype more in detail. K19-positive HCCs exhibited a significantly higher expression of miR-141, miR-200c, miR199a-3p, miR-218, miR-429 and miR-214 than K19-negative HCCs, whereas, miR-122, miR-885-5p, miR-148a, miR-519a, miR-148b, miR-556-5p, miR-193b, miR-618, miR-548b-3p, miR$10 \mathrm{~b}$ and miR-566 were relatively underexpressed in K19-positive HCCs (figure 5A).

The baseline expression levels of the most highly upregulated miRNAs, miR-141 and miR-200c, and the most downregulated, miR-885-5p and miR-122, were analysed in the HCC cell lines Huh-7D12, HepG2 and PLC/PRF/5 by qPCR. The members of the miR-200 family (miR-141/miR-200c) were strongly linked with KRT19 expression, in particular, miR-141 (figure 5B). To investigate the effect of these specific miRNAs on KRT19 
A

\begin{tabular}{|c|c|c|c|c|}
\hline \multicolumn{5}{|c|}{ Gene-expression analysis } \\
\hline Gene & $\begin{array}{c}\text { Correlation } \\
\text { (Pearson) }\end{array}$ & Description & $\begin{array}{c}\text { Gene } \\
\text { expressio } \\
n(n \text {-fold })\end{array}$ & p-value \\
\hline \multicolumn{5}{|c|}{ Progenitor cell markers } \\
\hline KRT19 & 1.00 & keratin 19 & +973 & 0.002 \\
\hline KRT7 & 0.53 & keratin 7 & +28 & 0.020 \\
\hline PROM1 & 0.69 & prominin 1 (CD133) & +8 & 0.007 \\
\hline GSTP1 & 0.60 & glutathione S-transferase pi & +7 & 0.021 \\
\hline \multicolumn{5}{|c|}{ Hepatocyte features } \\
\hline HNF4A & -0.51 & hepatocyte nuclear factor 4 , alpha & -2 & 0.007 \\
\hline$A B C C 2$ & -0.59 & ATP-binding cassette, sub-family $C$, member 2 & -5 & 0.021 \\
\hline \multicolumn{5}{|c|}{ Notch Pathway } \\
\hline$\overline{J A G 1}$ & 0.52 & jagged 1 (Alagille syndrome) & +7 & 0.004 \\
\hline JAG2 & - & jagged 2 & +3 & 0.030 \\
\hline NOTCH1 & - & notch, drosophila, homolog of, 1 & +2 & 0.020 \\
\hline NOTCH2 & - & notch, drosophila, homolog of, 2 & +4 & 0.032 \\
\hline NOTCH3 & - & notch, drosophila, homolog of, 3 & +4 & 0.005 \\
\hline \multicolumn{5}{|c|}{ Invasion-/Metastasis- related Markers } \\
\hline СТBP2 & 0.73 & C-terminal binding protein 2 & +4 & 0.007 \\
\hline S100A6 & 0.73 & S100 calcium binding protein $A 6$ (calcyclin) & +21 & 0.021 \\
\hline VASP & 0.67 & Vasodilator-stimulated phosphoprotein & +3 & 0.025 \\
\hline FAM57A & 0.63 & family with sequence similarity 57 member $A$ & +3 & 0.004 \\
\hline TACSTD2 & 0.63 & tumour-associated calcium signal transducer 2 & +7 & 0.008 \\
\hline ANXA3 & 0.65 & $\operatorname{annexin} A 3$ & +48 & 0.020 \\
\hline RAB25 & 0,65 & RAB25, member RAS oncogene family & +165 & 0.001 \\
\hline$R A B 27 B$ & 0.57 & Ras associated protein $27 \mathrm{~B}$ & +9 & 0.021 \\
\hline MTSS1 & -0.70 & metastasis suppressor 1 & -4 & 0.002 \\
\hline PDGFRA & . & platelet-derived growth factor receptor, alpha & +8 & 0.004 \\
\hline \multicolumn{5}{|c|}{ Extracellular matrix related } \\
\hline LAMB1 & 0.58 & laminin, beta 1 & +6 & 0.042 \\
\hline LAMC2 & 0.63 & laminin, gamma 2 & +8 & 0.021 \\
\hline \multicolumn{5}{|c|}{ Cytoskeleton } \\
\hline IQGAP1 & 0,58 & IQ motif containing GTPase activating protein 1 & +6 & 0.001 \\
\hline IQGAP2 & $-0,66$ & IQ motif containing GTPase activating protein 2 & -5 & 0.001 \\
\hline
\end{tabular}

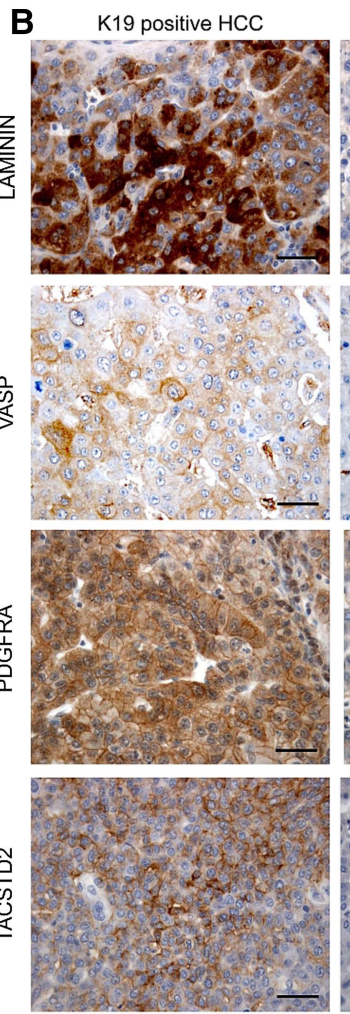

K19 negative HCC
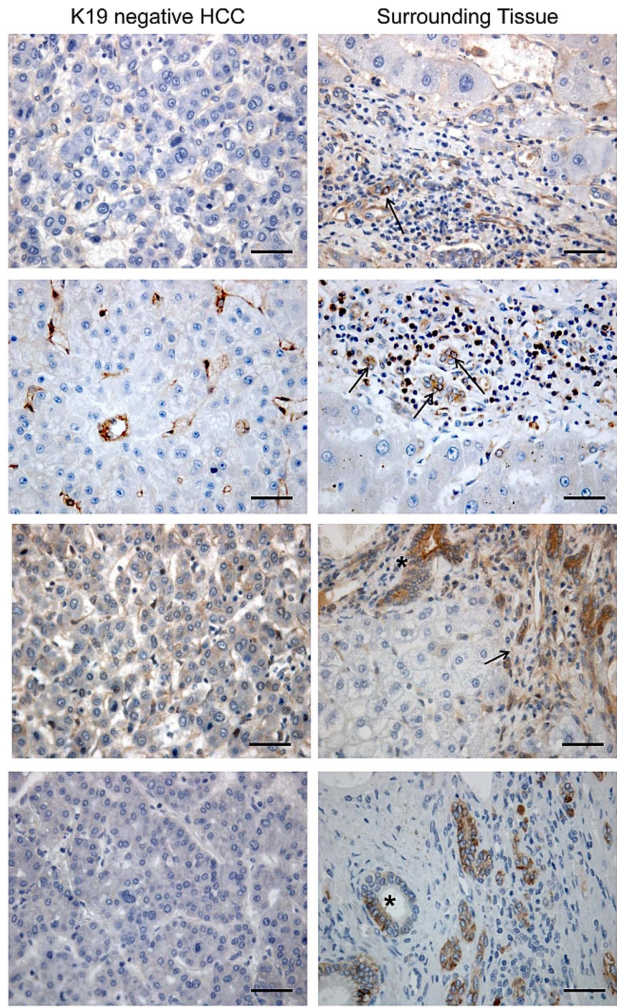

Figure 2 Molecular phenotype of K19-positive hepatocellular carcinomas (HCCs). (A) qPCR validation for a selected number of genes on K19-positive $(n=6)$ and -negative $(n=6)$ HCCs. Genes were selected based on the Pearson correlation coefficient and/or pathway association. Results are depicted as fold regulation ( $n$-fold) of K19-positive versus K19-negative samples $\left({ }^{* *} p<0.01 ;{ }^{*} p<0.05\right)$. (B) Immunohistochemical staining for laminin, VASP, PDGFRA and TACSTD2 (scale bars $100 \mu \mathrm{m}$ ). Left panel shows a representative example of a K19-positive HCC, middle panel a K19-negative HCC, right panel shows the surrounding tissue with extensive hepatic progenitor cell (HPC) activation (HPC, arrow; bile duct, asterisk).

mRNA expression and KRT19-related genes, miR-141/ miR-200c mimics were induced in the PLC/PRF/5, a K19-negative/KRT19-low HCC cell line. Transiently inducing the expression of miR-141and miR-200c significantly upregulated the expression of LAMC2, KRT19, KRT7, EPCAM, paralleling gene expression in K19-positive HCCs themselves, and significantly reduced the expression of hepatocyte-specific markers $A L B$ and HNF4A $(\mathrm{p}<0.01)$ when compared with the negative control (figure $5 \mathrm{C}$ ).

In situ hybridisation confirmed the strong link between miR-141 and K19 expression in human HCC samples and surrounding tissue. Additional in situ hybridisation for the most differentially upregulated miRNAs can be found in online supplementary file 5 .

\section{DISCUSSION}

In this present study, the clinicopathological relevance of K19 was assessed in a prospective Caucasian series of 242 consecutive HCC samples, consisting of 167 surgical specimens and 75 needle biopsies. As reported previously in a South Korean HCC cohort consisting mainly of samples from hepatitis B virus-infected patients, we were able to confirm the prognostic value of K19 expression in our Caucasian cohort with different underlying aetiologies as it significantly correlated with tumour size, decreased tumour differentiation, metastasis and microvascular invasion. Despite the possible overestimation or underestimation of protein expression, we confirmed the prognostic value of K19 expression in diagnostic needle biopsies. ${ }^{4}$ In this study, EPCAM did not prove to have the same prognostic value as K19. This could be explained by the fact that in regenerating liver, EPCAM is a much broader marker than $\mathrm{K} 19$, as it also marks the progeny of the HPCs, the intermediate hepatocytes, similar to $\mathrm{K} 7 .^{20}$

In order to better understand the more aggressive behaviour of K19-positive HCCs, we unravelled the underlying molecular phenotype. The KRT19-associated gene signature showed a strong overlap with that of other previously described more malignant HCC subclasses, such as 'poor survival HCC subtype', 'proliferation HCC subtype' and 'subtype S1 signature with aberrant Wnt activation'. ${ }^{14} 2122$ In addition to the occurrence of other HPC/biliary markers (eg, PROM1/CD133, GSTP1, KRT7), K19-positive HCCs expressed a whole range of invasion-related/metastasis-related genes, of which some proved to be present in the surrounding non-neoplastic HPCs (eg, TACSTD2, VASP, PDGFRA). ${ }^{12} 23^{24}$ To date, it is not clear whether the expression of HPC markers in human HCCs is a result of dedifferentiation of malignant hepatocytes during continuous mutagenesis, or whether this is the conserved 'fingerprint' of a HPC tumour origin. Direct evidence for the involvement of HPCs in the histogenesis of HCC has been found by Dumble et al, by isolating HPCs from p53 null mice. $^{25}$ In human chronic liver disease, our group described that about half the preneoplastic small-cell dysplastic foci consisted almost completely of cells that have the immunophenotypic profile of HPCs and intermediate hepatocyte-like cells, suggesting that HPCs can give rise to HCCs in human liver. ${ }^{26}$ In this present study, the expression of similar markers in HPCs and K19-positive HCCs is compatible with a HPC origin. The aberrant expression of the Notch pathway at least insinuates that HPCs and K19-positive HCC cells share some common 
A
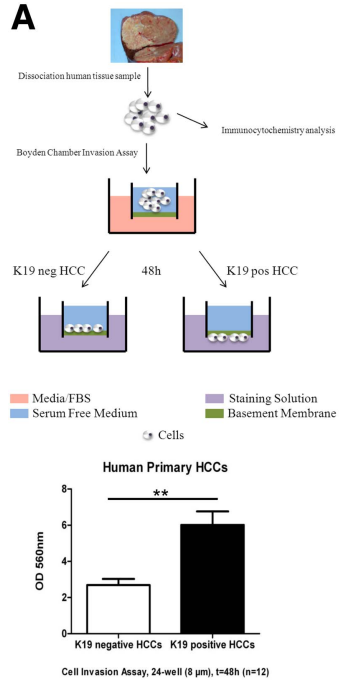

C
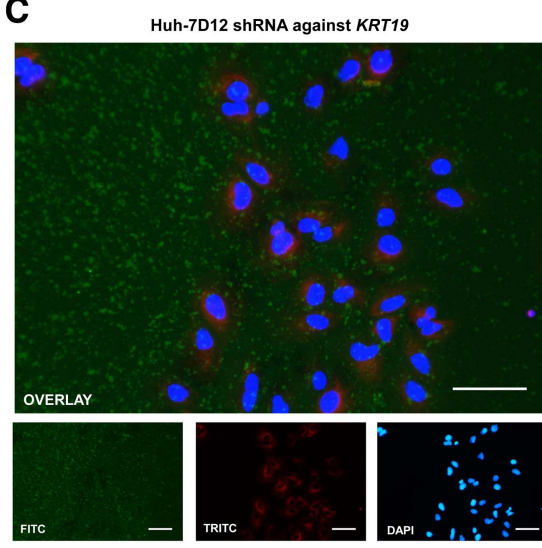
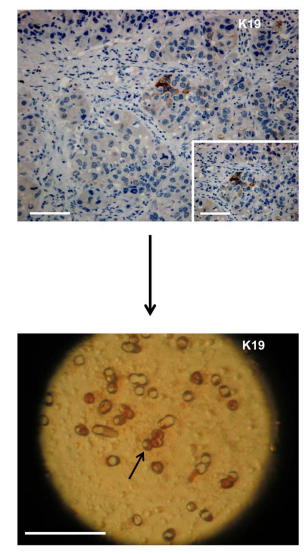

B

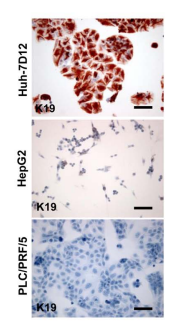

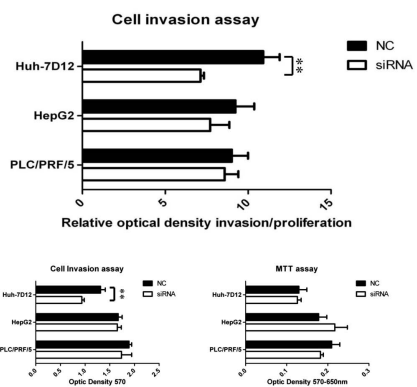

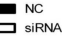
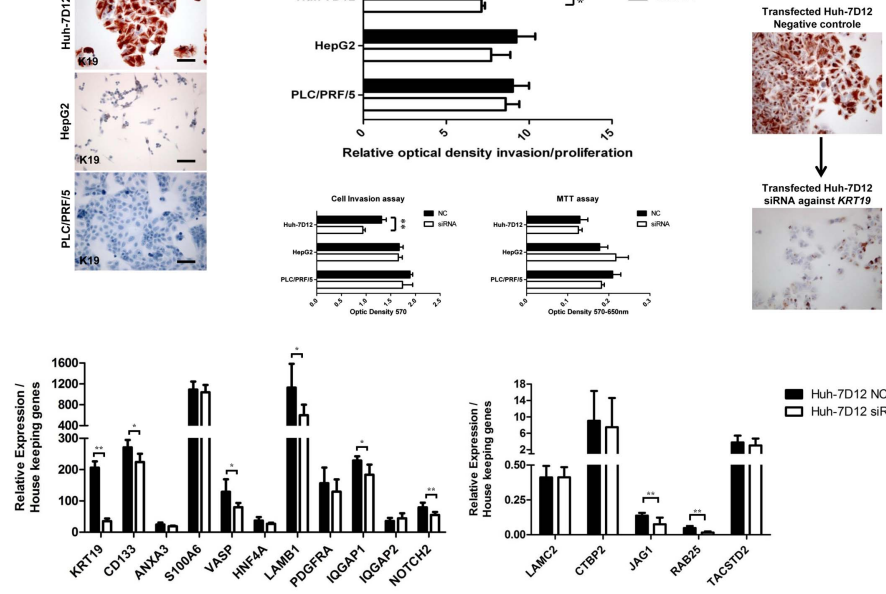

Huh-7D12 NC 工南

Figure 3 A role for keratin 19 in tumour invasion. The ability of tumour cells to invade is one of the hallmarks of the metastatic phenotype. (A) Human dissociated hepatocellular carcinomas (HCC) were subjected to an extracellular matrix (ECM) cell invasion assay. After $48 \mathrm{~h}$, cells from human primary K19-positive HCCs showed a higher invasiveness than cells from K19-negative HCCs ( $<0.01 ; n=12)$. Immunostaining showed an increase in K19-positive tumour cells after invasion, as compared with the original tumour. (B) Huh-7D12, PLC/PRF/5 and HepG2 HCC cell lines were characterised by means of immunocytochemistry (left panel, scale bars $100 \mu \mathrm{m}$ ). After $72 \mathrm{~h}$, the MTT assay showed no significant differences in proliferation between KRT19/K19 knockdown and control $(n=3)$; the knockdown only significantly reduced relative invasiveness of Huh-7D12 with 34.7\%. Immunohistochemistry for K19 shows the decline of expression in transfected Huh-7D12 cells (scale bars $100 \mu \mathrm{m}$ ). The effect of the transient knockdown on the expression of KRT19-associated genes was done by qPCR (displayed as relative fold expression) $\left({ }^{* *} p<0.01\right.$; $\left.{ }^{*} p<0.05\right)$. (C) Stably transduced Huh-7D12 cells were submitted to a QCM Gelatin Invadopodia Assay (24 h). The transduced cells were seeded onto green fluorescein-conjugated gelatin substrates and after $24 \mathrm{~h}$ visualised with wide-spectrum cytokeratin (red) and DAPI (blue). Gelatin degradation demonstrated that both the KRT19 knockdown as the negative control still possess invasive capacity, although cells transduced with a construct against KRT19 showed less intense positivity for cytokeratin wide-spectrum screening (left panel, scale bars $100 \mu \mathrm{m}$ ). Invaded cells with reduced KRT19/K19 expression demonstrated a decreased formation of invadopodia and regular cell morphology, compared with the negative control (right panel, scale bars $50 \mu \mathrm{m})$.

activated pathways. ${ }^{27}$ Our recent insights in the role of Notch signalling in liver regeneration showed that activation of the Notch pathway is pivotal for differentiation towards biliary cell fate. ${ }^{28}$ Additionally, a strong cytoplasmic expression of laminin was observed in K19-positive HCCs, as noted in areas of nonneoplastic HPCs in the surrounding tissue. In human liver disease, laminin has been reported to be part of the basement membrane surrounding the HPCs in order to help them remain in an undifferentiated state, indicating that K19-positive HCCs might produce laminin to maintain their 'stemness'. ${ }^{29}$ MicroRNA profiling and functional validation in cell lines, likewise, illustrated how members of the miR-200 family (eg, miR-141/miR-200c) are strongly linked with K19/KRT19 expression. In vitro modulation of these particular miRNAs in a
K19-negative/KRT19-low HCC cell line actually induced the expression of biliary/HPC markers and, moreover, the expression of $L A M C 2$. It is possible that miR-141 and miR-200c contribute to the HPC microenvironment and 'stemness' through modulation of laminin.

K19-positive HCCs express more invasion-related/metastasisrelated genes compared with the K19-negative HCCs, and they also showed an increased invasive capacity in vitro. Moreover, keratin 19 positive tumour cells, in particular, exhibited the highest invasive capacity. The role of the cytoskeleton and associated proteins as cellular integrators in the neoplastic process has already been suggested back in $1985 .^{30}$ Our findings demonstrate functional consequences of K19 expression, since KRT19 knockdown significantly reduced HCC invasive ability in vitro and 
A

\section{Size of Side Population in primary HCC samples}
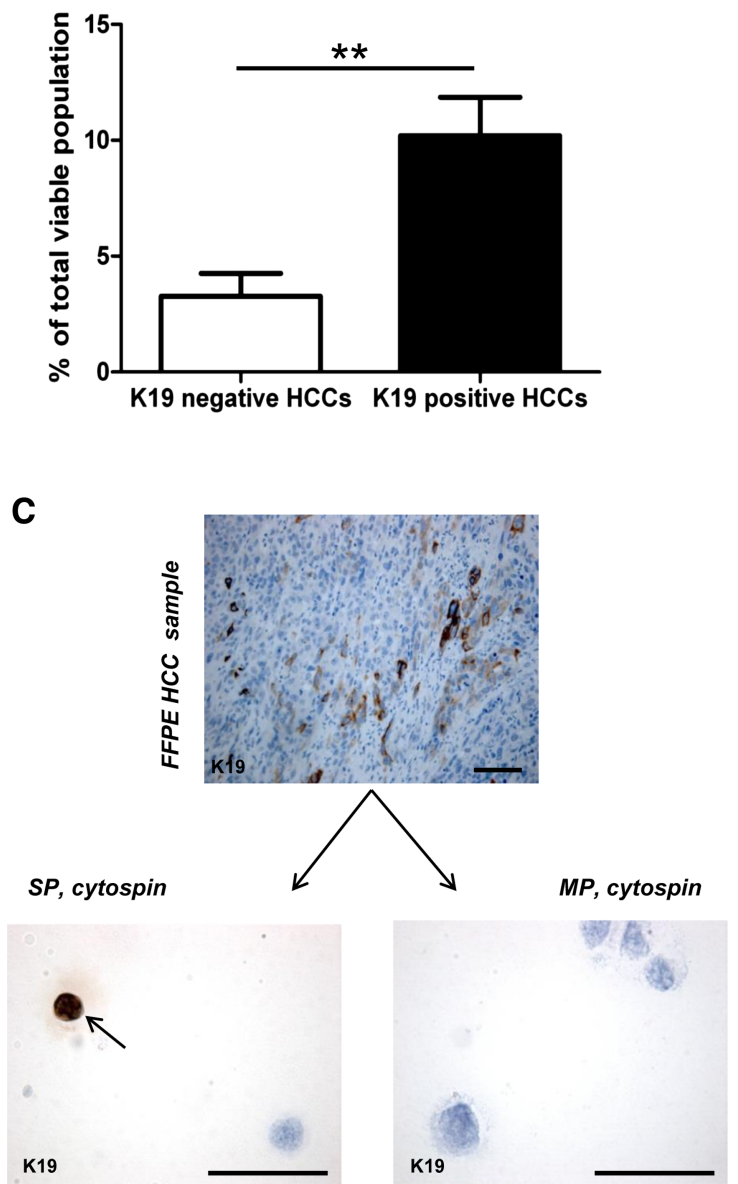

B
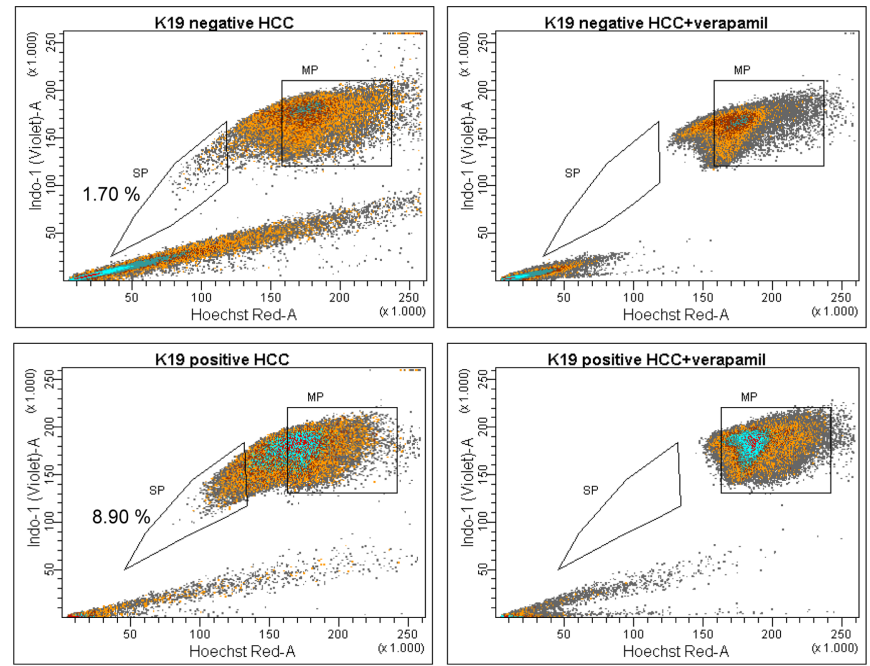

D

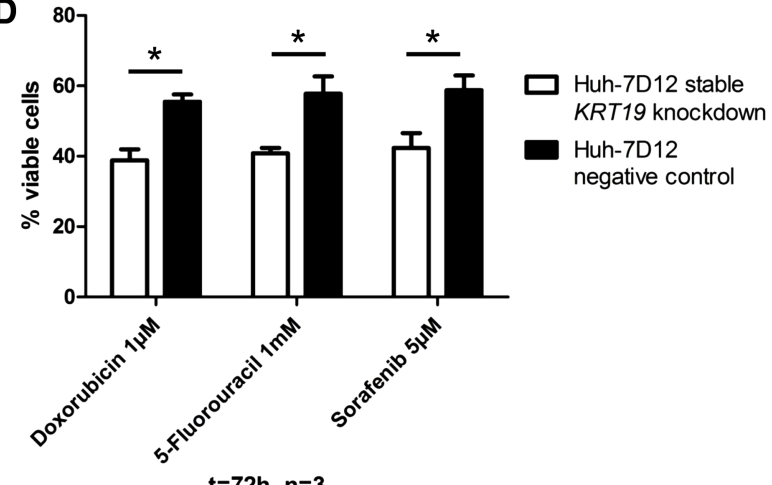

$t=72 h, n=3$

Figure 4 Keratin 19 and treatment resistance. Side population (SP) is based on the ability of stem-like cells to efflux the fluorescent DNA-binding dye Hoechst 33342. The phenotype was obtained using the ATP-binding cassette-transporter blocker verapamil. (A) Using flow cytometric analysis, a SP was cell sorted from the rest of the main population (MP), differing from an average of $3.3 \%( \pm 2.4)$ in human K19-negative hepatocellular carcinomas (HCC) $(n=6)$ to $10.2 \%( \pm 4.1)$ in human K19-positive HCCs $(n=6)$ of the total viable cells. (B) A representative example of the SP plot of a human K19-positive and K19-negative HCC. (C) Immunocytochemistry on cytospins of sorted cells showed that all the K19-positive cells were located in the SP (scale bar formalin-fixed paraffin-embedded (FFPE) sample $200 \mu \mathrm{m}$; scale bars cytospins $100 \mu \mathrm{m}$ ). (D) Stably transduced Huh-7D12 cells were treated with either $1 \mu \mathrm{M}$ doxorubicin, $1 \mathrm{mM}$ fluorouracil or $5 \mu \mathrm{M}$ sorafenib $(\mathrm{n}=3)$ for $72 \mathrm{~h}$. The effect was measured compared with untreated cells, using a MTT assay, and displayed as \% viable cells. Cells stably transduced against KRT19/K19 show a decreased viability when treated for the selected agents as compared with the negative control ( $\left.{ }^{*} p<0.05\right)$.

compromised the formation of invadopodia. A possible explanation could be found in the influence of K19 on VASP and LAMB1. VASP, a regulator of filopodia formation, has been reported to play a role in adhesion dynamics and migration by actively linking the cytoskeleton to the ECM in an integrin-dependent manner, whereas $L A M B 1$, known to interact with integrin receptors, has been associated with tumour invasion. ${ }^{31} 32$ Considering the effect of K19 on these specific genes, we hypothesise that K19 is a key element in the transmembrane crosstalk between the cytoskeleton, filopodia formation and the ECM.

Resistance to chemotherapy is commonly seen in the treatment of HCC and is associated with cancer relapse and metastasis. Multidrug resistance is frequently due to high $\mathrm{ABC}$ transporter expression, enabling resistant cells to efflux potentially harmful substances; thereby conferring a survival advantage. SP analysis is an isolation technique that differentiates cell populations based on $\mathrm{ABC}$ transporter function. ${ }^{33}$ This technique was first reported by Goodell et $a l^{34}$ for the isolation of murine haematopoietic stem cells with long-term multilineage repopulating potential. In human HCC cell lines, SP isolation has proven to be a useful approach to sort cells with a 'stemness' signature with increased tumour-initiation capacity and a higher resistance to cytotoxic agents (eg, doxorubicin, 5-fluorouracil and gemcitabine) compared with the non-SP fraction. ${ }^{19} \quad 35 \quad 36$ These stem cell-like cancer cells are believed to provide heterogeneity to the cancer cell system. This cancer stem cell concept has enormous implications for cancer therapy. Most treatments target the rapidly dividing differentiating cells that comprise the major bulk of tumours, often leading to significant reduction in tumour size, although without eliminating the driving force in tumour progression, the resistant cancer stem cells. In this study, the SP fraction was significantly larger in the K19-positive HCCs compared with K19-negative HCC, and, most importantly, contained all the cells expressing K19. This 
A Differentially expressed microRNAs Upregulated

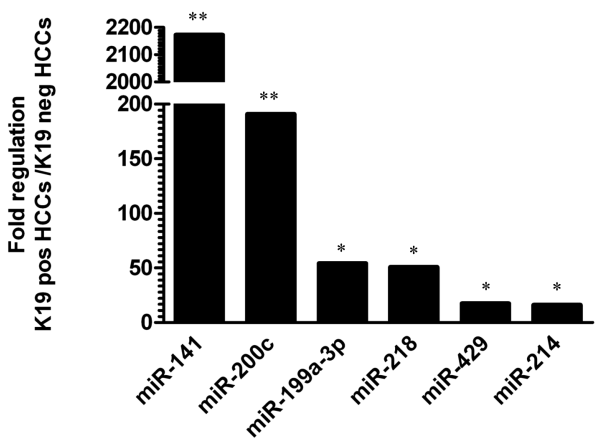

Differentially expressed microRNAs Downregulated

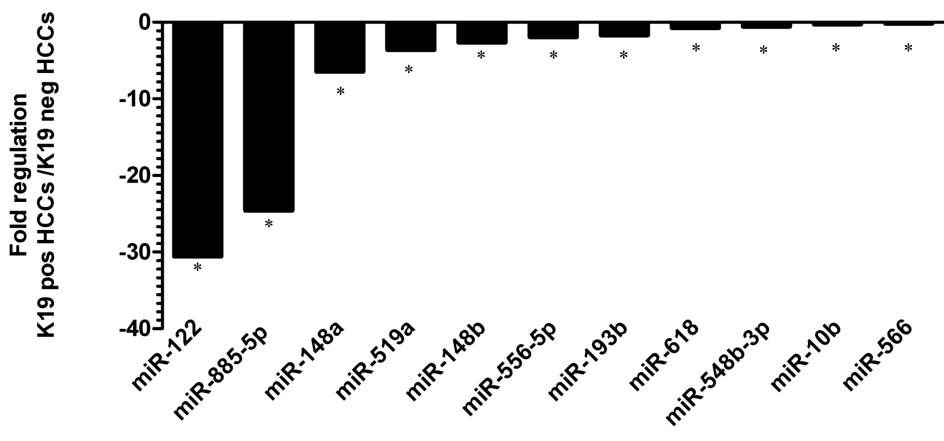

C
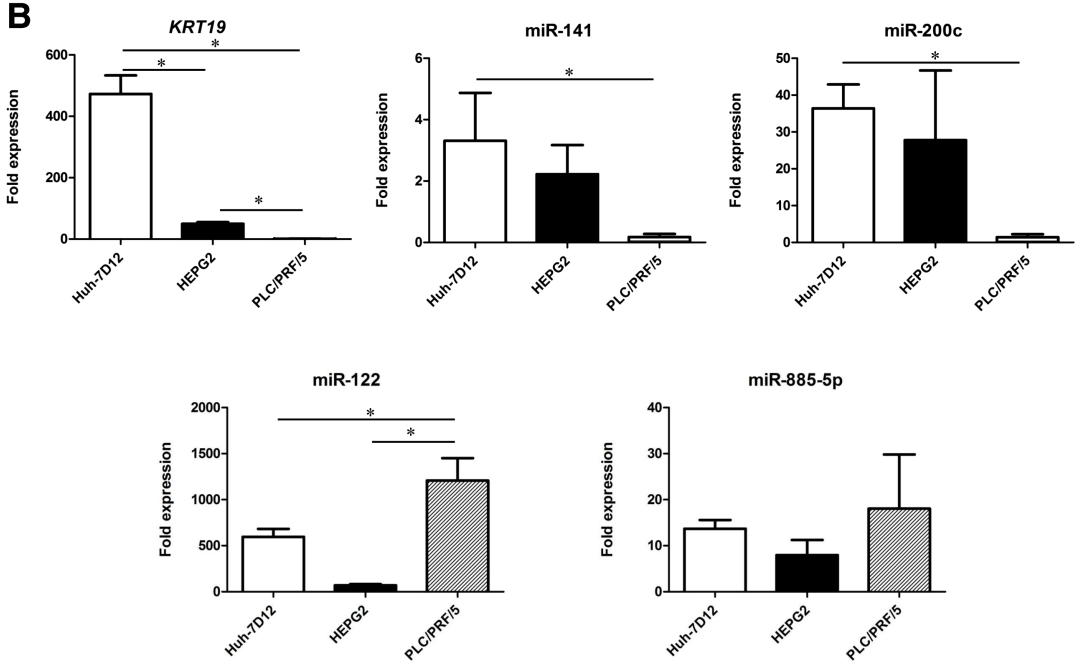

D
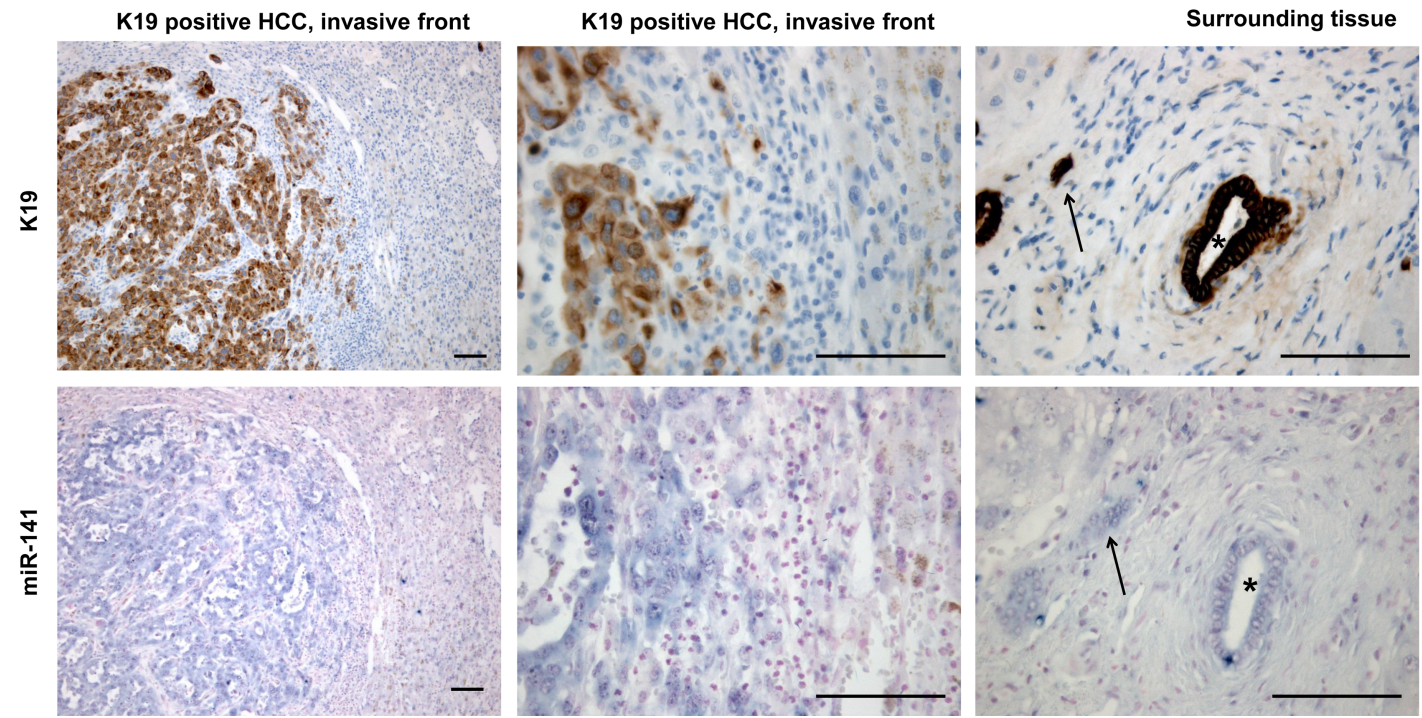

Figure 5 MicroRNA profiling of K19-positive hepatocellular carcinomas (HCC). (A) MiRNA profiling of K19-positive ( $n=6)$ and -negative HCCs $(n=6)$. Results are depicted as fold regulation ( $n$-fold) of K19-positive versus K19-negative samples $\left({ }^{* *} p<0.01\right.$; $\left.{ }^{*} p<0.05\right)$. (B) Characterisation of Huh-7D12, PLC/PRF/5 and HepG2 HCC cell lines for KRT19 and the most differentially expressed miRNAs found in the human samples (eg, hsa-miR-141, hsa-miR-200c, hsa-miR-122 and has-miR-885-5p) was done by means of qPCR. Results are depicted as fold expression compared to housekeeping genes/nuclear microRNAs $\left({ }^{*} p<0.05\right)$. (C) In vitro modulation of the function of hsa-miR-141 and hsa-miR-200c was done by inducing synthetic mimic locked nucleic acid probes in the K19 negative PLC/PRF/5 HCC cell line. Target read-out of the induced miRNA expression was done by means of $q P C R$ and the data is presented as a $\log 2$ scale of the fold regulation of mimic/hairpin to negative control $\left({ }^{* *} p<0.01 ;{ }^{*} p<0.05\right)$. (D) Immunohistochemistry and in situ hybridisation on human HCC formalin-fixed paraffin-embedded samples for keratin 19 and miR-141. Left panel shows the invading front of a K19-positive HCC, middle panel shows the center bulk of the K19-positive HCC, right panel shows the surrounding tissue. Scale bars $200 \mu \mathrm{m}$. miR-141 expression strongly correlated with K19 expression in HCCs, and in the bile ducts (asterisk)/hepatic progenitor cells (arrow) of the surrounding. 
could explain why K19 immunopositivity in HCC is clinically relevant starting from only five per cent cell positivity, as those K19-positive cells may be responsible for the chemoresistant/ stemness phenotype in these cancers. ${ }^{7}$ Moreover, disrupting the HPC/biliary phenotype by diminishing K19/KRT19 expression, rendered HCC cells more sensitive to cytotoxic agents as doxorubicin, fluorouracil and sorafenib in vitro.

K19 by itself is neither an oncogene (as it is expressed in nonmalignant cells as well) nor is it a protein directly involved in chemoresistance, but our data clearly showed that K19 is a critical player in the invasive and resistant behaviour of these subtypes of HCCs. Additional partners are probably necessary to support this specific function of K19. VASP and LAMB1 are potential candidates, but also the presence of PDGFRA provides us a possible explanation for the increased malignancy of K19-positive HCCs. Several PDGFRA activation mutations have been described to contribute to tumour initiation and progression. Identification and further understanding of K19-associated genes might open horizons for a more patient-specific treatment, for example, PDGFRA targeting by treatment with imatinib. ${ }^{24}$

Viewing the different molecular and epigenetic profile as well as the distinct invasive properties of K19-positive HCCs, this subclass of HCCs with adverse clinical outcome should be considered as a separate entity from the K19-negative HCCs in daily clinical practice. Diagnostic pathology based on immunohistochemistry offers a fast, cheap and widely accessible method to recognise this particular subclass of HCCs.

\footnotetext{
Author affiliations

${ }^{1}$ Department of Imaging and Pathology, KU Leuven and University Hospitals Leuven, Leuven, Belgium

${ }^{2}$ Dipartimento Oncologico AUSL 4, Institute Toscano Tumori (ITT), Prato, Italy ${ }^{3}$ Department of Development and Regeneration, KU Leuven, Leuven, Belgium ${ }^{4}$ Department of Pathology, McGill University/Jewish General Hospital, Montreal, Quebec, Canada

${ }^{5}$ Department of Hepatology, KU Leuven and University Hospitals Leuven, Leuven, Belgium

${ }^{6}$ Department of In Vitro Toxicology and Dermato-cosmetology (IVTD/FAFY), Vrije Universiteit Brussel, Brussels, Belgium

${ }^{7}$ Department of Cell Biology, Liver Cell Biology Lab, Vrije Universiteit Brussel, Brussels, Belgium

${ }^{8}$ Department of Abdominal Surgery, KU Leuven and University Hospitals Leuven, Leuven, Belgium

${ }^{9}$ Department of Abdominal Transplant Surgery, KU Leuven and University Hospitals Leuven, Leuven, Belgium

${ }^{10}$ University College London, Institute for Liver and Digestive Health, Royal Free Hospital, London, UK
}

Acknowledgements The authors would like to thank Prof Dr Valeer I Desmet, Prof Dr Raymond Aerts, Prof Dr Evelyne Lerut, Dr Katharine Irvine, Dr Sara Vander Borght, Kathleen Van den Eynde, Paula Aertsen, Miet Vanherck and Frank Vanderhoydonc for their indispensable support.

Contributors OG: study concept and design; acquisition of data; analysis and interpretation of data; drafting of the manuscript; statistical analysis. MK, JB and BS study concept and design; acquisition of data; analysis and interpretation of data.CJ, FdL, JW, AD, CV, JD K and JP: acquisition of data.AK: acquisition of data; statistical analysis.LCvK: study concept and design; acquisition of data.VR, LAvG, HV and FN: study concept and design.BT: material support.JvdO: critical revision of the manuscript for important intellectual content; obtained funding; study supervision. MP: study concept and design; critical revision of the manuscript for important intellectual content; obtained funding; study supervision. TR: study concept and design; analysis and interpretation of data; critical revision of the manuscript for important intellectual content; obtained funding; study supervision.

Funding This study was supported by a grant of the Interuniversity Attraction Poles (IAP) Programme of the Belgian Science Policy Office (Phase VI and VII: HEPRO I and II).

Competing interests None.

Ethics approval The ethical committee of the University Hospitals Leuven, Belgium.

Provenance and peer review Not commissioned; externally peer reviewed.
Data sharing statement The authors state to make required materials, data and associated protocols promptly available when requested.

Open Access This is an Open Access article distributed in accordance with the Creative Commons Attribution Non Commercial (CC BY-NC 3.0) license, which permits others to distribute, remix, adapt, build upon this work non-commercially, and license their derivative works on different terms, provided the original work is properly cited and the use is non-commercial. See: http://creativecommons.org/ licenses/by-nc/3.0/

\section{REFERENCES}

1 Roskams T. Liver stem cells and their implication in hepatocellular and cholangiocarcinoma. Oncogene 2006;25:3818-22.

2 Zhuang PY, Zhang J-B, Zhu X-D, et al. Two pathologic types of hepatocellula carcinoma with lymph node metastasis with distinct prognosis on the basis of CK19 expression in tumor. Cancer 2008;112:2740-8

3 Uenishi T, Kubo S, Yamamoto T, et al. Cytokeratin 19 expression in hepatocellular carcinoma predicts early postoperative recurrence. Cancer Sci 2003;94:851-7.

4 Tsuchiya K, Komuta M, Yasui Y, et al. Expression of keratin 19 is related to high recurrence of hepatocellular carcinoma after radiofrequency ablation. Oncology 2011:80:278-88

5 Yang XR, Xu Y, Shi G-M., et al. Cytokeratin 10 and cytokeratin 19: predictive markers for poor prognosis in hepatocellular carcinoma patients after curative resection. Clin Cancer Res 2008;14:3850-9.

6 . Kim H, Choi GH, Na DC, et al. Human hepatocellular carcinomas with "Stemness"-related marker expression: keratin 19 expression and a poor prognosis. Hepatology 2011;54:1707-17.

7 Durnez A, Verslype C, Nevens F, et al. The clinicopathological and prognostic relevance of cytokeratin 7 and 19 expression in hepatocellular carcinoma. A possible progenitor cell origin. Histopathology 2006;49:138-51.

8 Roskams T, Katoonizadeh A, Komuta M. Hepatic progenitor cells: an update. Clin Liver Dis 2011;14:705-18.

9 Komuta M, Spee B, Borght SV, et al. Clinicopathological study on cholangiolocellular carcinoma suggesting hepatic progenitor cell origin. Hepatology 2008;47:1544-56.

10 Vander Borght S, Komuta M, Libbrecht L, et al. Expression of multidrug resistance-associated protein 1 in hepatocellular carcinoma is associated with a more aggressive tumour phenotype and may reflect a progenitor cell origin. Liver Int 2008;28:1370-80.

11 Fletcher $\mathrm{Jl}$, Haber $\mathrm{M}$, Henderson $\mathrm{M}$, et al. $\mathrm{ABC}$ transporters in cancer: more than just drug efflux pumps. Nat Rev Cancer 2010;10:147-56.

12 Komuta M, Govaere 0, Vandecaveye V, et al. Histological diversity in cholangiocellular carcinoma reflects the different cholangiocyte phenotypes. Hepatology 2012;55:1876-88.

13 van Kempen LC, van Muijen GN, Ruiter DJ. Melanoma progression in a changing environment. Eur J Cell Biol 2007;86:65-7.

14 Lee JS, Chu I-S, Heo J, et al. Classification and prediction of survival in hepatocellular carcinoma by gene expression profiling. Hepatology 2004:40:667-76

15 Lee JS, Heo J, Libbrecht L, et al. A novel prognostic subtype of human hepatocellular carcinoma derived from hepatic progenitor cells. Nat Med 2006:12:410-6.

16 Subramanian A, Tamayo P, Mootha VK, et al. Gene set enrichment analysis: a knowledge-based approach for interpreting genome-wide expression profiles. Proc Natl Acad Sci USA 2005;102:15545-50.

17 Mootha VK, Lindgren CM, Eriksson K-F, et al. PGC-1alpha-responsive genes involved in oxidative phosphorylation are coordinately downregulated in human diabetes. Nat Genet 2003:34:267-73.

18 Szklarczyk D, Franceschini A, Kuhn M, et al. The STRING database in 2011: functional interaction networks of proteins, globally integrated and scored. Nucleic Acids Res 2011;39:D561-568.

19 Haraguchi $\mathrm{N}$, Utsunomiya $\mathrm{T}$, Inoue $\mathrm{H}$, et al. Characterization of a side population of cancer cells from human gastrointestinal system. Stem Cells 2006;24:506-13.

20 Yoon SM, Gerasimidou D, Kuwahara R, et al. Epithelial cell adhesion molecule (EpCAM) marks hepatocytes newly derived from stem/progenitor cells in humans. Hepatology 2011;53:964-73.

21 Chiang DY, Villanueva A, Hoshida Y, et al. Focal gains of VEGFA and molecula classification of hepatocellular carcinoma. Cancer Res 2008;68:6779-88.

22 Hoshida Y, Nijman SMB, Kobayashi M, et al. Integrative transcriptome analysis reveals common molecular subclasses of human hepatocellular carcinoma. Cancer Res 2009:69:7385-92.

23 Dertsiz L, Ozbilim G, Kayisli Y, et al. Differential expression of VASP in normal lung tissue and lung adenocarcinomas. Thorax 2005:60:576-81.

24 Oseini AM, Roberts LR. PDGFRalpha: a new therapeutic target in the treatment of hepatocellular carcinoma? Expert Opin Ther Targets 2009;13:443-54.

25 Dumble ML, Croager EJ, Yeoh GC, et al. Generation and characterization of p53 null transformed hepatic progenitor cells: oval cells give rise to hepatocellular carcinoma. Carcinogenesis 2002:23:435-45. 
26 Libbrecht L, Desmet V, Roskams T. Preneoplastic lesions in human hepatocarcinogenesis. Liver Int 2005;25:16-27.

27 Forbes SJ, Alison MR. Side population (SP) cells: taking center stage in regeneration and liver cancer? Hepatology 2006;44:23-6.

28 Boulter L, Govaere O, Bird TG, et al. Macrophage-derived Wnt opposes Notch signaling to specify hepatic progenitor cell fate in chronic liver disease. Nat Med 2012;18:572-9.

29 Lorenzini S, Bird TG, Boulter L, et al. Characterisation of a stereotypical cellular and extracellular adult liver progenitor cell niche in rodents and diseased human liver. Gut 2010:59:645-54

30 Bernal SD, Stahel RA. Cytoskeleton-associated proteins: their role as cellular integrators in the neoplastic process. Crit Rev Oncol Hematol 1985:3:191-204.

31 Worth DC, Hodivala-Dilke K, Robinson SD, et al. Alpha v beta3 integrin spatially regulates VASP and RIAM to control adhesion dynamics and migration. J Cell Biol 2010;189:369-83.
32 Givant-Horwitz V, Davidson B, Reich R. Laminin-induced signaling in tumor cells: the role of the $M(r) 67,000$ laminin receptor. Cancer Res 2004;64:

3572-9.

33 Alison MR, Guppy NJ, Lim SM, et al. Finding cancer stem cells: are aldehyde dehydrogenases fit for purpose? J Pathol 2010;222:335-44.

34 Goodell MA, Brose K, Paradis G, et al. Isolation and functional properties of murine hematopoietic stem cells that are replicating in vivo. J Exp Med 1996;183:1797-806.

35 Chiba T, Kita K, Zheng Y-W, et al. Side population purified from hepatocellular carcinoma cells harbors cancer stem cell-like properties. Hepatology 2006;44:240-51

36 Marquardt JU, Raggi C, Andersen JB, et al. Human hepatic cancer stem cells are characterized by common stemness traits and diverse oncogenic pathways. Hepatology 2012;54:1031-42. 